\title{
Paul Summergrad, MD, on the state of psychiatry
}

\author{
Awais Aftab, MD, and Paul Summergrad, MD
}

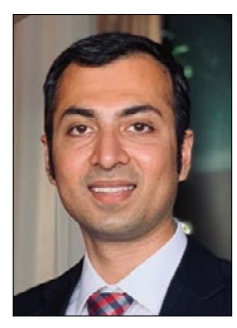

Awais Aftab, MD

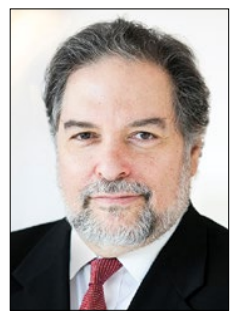

Paul Summergrad, MD

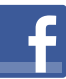

Discuss this article at www.facebook.com/ MDedgePsychiatry or this Psychiatry Leaders' Perspectives, Awais Aftab, MD, interviewed Paul Summergrad, MD. Dr. Summergrad is the Dr. Frances S. Arkin Professor and Chair of the Department of Psychiatry and Professor of Psychiatry and Medicine at Tufts University School of Medicine and Psychiatrist-in-Chief at Tufts Medical Center, Boston, Massachusetts. From 2014 to 2015, Dr. Summergrad served as the 141st president of the American Psychiatric Association, and is a past president of the American Association of Chairs of Departments of Psychiatry. Dr. Summergrad's research focuses on mood disorders, medical/psychiatric comorbidity, and health system design. He received the Distinguished Faculty Award from Tufts University School of Medicine in 2015 and the Leadership Award of the American Association of Chairs of Departments of Psychiatry in 2018. In 2020, he was elected to the Honorary Fellowship of the Royal College of Psychiatrists, their highest honor. He is the lead editor of Textbook of Medical Psychiatry, which was published by American Psychiatric Association Publishing in 2020.

Dr. Aftab: Much of your career has been devoted to the practice of "medical psychiatry" in which you have fruitfully integrated your medical training as well as psychoanalytic training. How has this influenced your understanding of the medical model in psychiatry and psychiatry's relationship with medicine?
Dr. Summergrad: It is a really complex and ongoing influence. I think there is a misunderstanding of what is meant by the medical model in psychiatry. It has nothing to do with the etiology of mental disorders or their treatment. At its core, the medical model is based on a syndromic view of disorders: that we attend to those symptoms of illness that occur together more frequently than they might by chance and then, based on that provisional diagnostic cluster, look for causes, risk factors, and interventions that affect the putative disorder's course. As a consequence of that process, disorders are refined, often separated into a group of disorders, or in some cases discarded. An excellent example that we have all been living through has been our evolving understanding of COVID-19, which is now understood to be as much a clotting and inflammatory disorder as a respiratory condition.

Medical psychiatry is a different and discrete area of clinical psychiatric interest. It covers a variety of areas: the complexity of the management of patients with comorbid medical and psychiatric illness, the impact of medical illness on the course of psychiatric illness and life expectancy, and

Dr. Aftab is Clinical Assistant Professor, Psychiatry, Case Western Reserve University, Cleveland, Ohio. Dr. Summergrad is the Dr. Frances S. Arkin Professor and Chair, Department of Psychiatry, and Professor of Psychiatry and Medicine, Tufts University School of Medicine; and Psychiatrist-in-Chief, Tufts Medical Center, Boston, Massachusetts. Dr. Summergrad is an Editorial Consultant for CURRENT PSYCHIATRY.

Disclosures

The authors report no financial relationships with any companies whose products are mentioned in this article, or with manufacturers of competing products.

doi: 10.12788/cp.0076 
conversely the effects of psychiatric illness on the course of medical disordersfor example, the increased mortality in patients with myocardial infarction (MI) and post-MI major depression. At its core is the study of medical disorders, including neurologic conditions, that directly cause syndromes in the realm that we define as mental disorders. This was the focus of our recent Textbook of Medical Psychiatry. This has been a long-standing interest of mine since I did my medical residency at Boston City Hospital before I trained in psychiatry, and it has informed my career in many other ways.

Dr. Aftab: What do you see as some of the strengths of our profession?

Dr. Summergrad: Psychiatry has so many riches: a long clinical tradition based on close and long-term observation and interaction with patients, effective psychotherapies such as cognitive-behavioral therapy and psychodynamic therapies, and evidence-based pharmacologic and other somatic therapies.

Second, there has been substantial growth in our fundamental understanding of the neurobiology of psychiatric illness with regards to circuitry, imaging, and genetics. While many of these advances have arisen from more basic research, it is also the case that the evolution of a consistent diagnostic nomenclature in the 1970s, even with all its limitations, allowed for advances in research, diagnosis, and treatment.

Finally, our other great strengths are our roots in medicine and the importance of those skills in assessing patients and caring for active comorbidities. We are one of the last fields in clinical medicine where doctors can take the time to establish a detailed and close working relationship with our patients. We are fortunate to have this great mix of science, medicine, and interpersonal skills, which is highly rewarding.
Dr. Aftab: Are there ways in which the status quo in psychiatry falls short of the ideal? What are our areas of relative weakness?

Dr. Summergrad: There are many, as there are in many other fields of medicine. For too many, there is a reification of a diagnostic nomenclature as being identical to detailed and thoughtful clinical evaluation. For many, the pressures of health care economics mean that they may be taking care of more inpatients than is optimal, or are under pressure to see a larger number of patients as a so-called "prescriber," a term I think should be banished.

We have struggled significantly to have a coherent link between our clinical work, including our interventions, our emerging understanding of neuroscience and genetics, and the experiences of our patients, including the onset and timing of many of the disorders we treat. Part of this is that we lack a unified model of mental functioning that unites the onset of illness, its clinical phenomena, and any underlying pathophysiology. We operate at multiple levels of abstraction (brain-mind) compared with other medical fields. While other medical fields incorporate experience, they are more fully operating, from a pathophysiologic perspective, at a physical level alone. Even in common parlance, we can easily talk about the heart as a pump, or the kidney as a filter, but there is no corresponding way to describe what the brain-mind is and does. This could be construed as a weakness; I see it more as an intrinsic complexity of our field.

What we refer to as psychiatric disorders deal with the most intimate aspects of people's beings: their sense of self and capacity. Many people experience our diagnostic work and nomenclature as wounding, demeaning, distancing, or defining their very essence or being as ill. There is a wonderful story that I heard from the great Elyn Saks, the constitutional law professor, regarding her own illness, about which she

\section{Clinical Point}

There has been substantial growth in our fundamental understanding of the neurobiology of psychiatric illness 


\section{Clinical Point}

\section{I don't think we}

have come near to tapping the effects of epigenetics on psychiatric illnesses has been admirably open. She described a long course of significant psychotic illness, eventually diagnosed as schizophrenia, for which she received years of psychotherapy, psychopharmacology, and hospital care, both when she studied at Oxford and while she was a law student at Yale. She described that after 10 years of care, she was eventually prescribed clozapine, which made a major difference in her illness. It was about the same time that she finally accepted that she had a psychiatric illness, and it was at that very moment of acceptance that she realized that it wasn't about her, that it didn't define who she was in her essence. Very moving and important. In defining pathophysiology or what we call psychopathology, we need to make sure it is clear that we are not labeling or diagnosing anyone's essential being.

I think we need to tread very carefully in these areas, including being very sensitive with our language. Much of this is in the nature of the illnesses we deal with and their profound intimacy, but again we need to be mindful of this. These areas are ones which I think contribute to a resentment of psychiatry, and are possibly related to some of the anti-psychiatry sentiments and criticisms of the so-called medical model in psychiatry, which as I noted above is, I think, not well understood.

Dr. Aftab: What is your perception of the threats that psychiatry faces or is likely to face in the future?

Dr. Summergrad: I actually am very bullish on psychiatry's future. While we are far from perfect, the illnesses we care for are so ubiquitous that many if not most people will experience them personally or in close family members over their lifetime. As such, there is a real and broad understanding about the need for psychiatric care; we do, however, have to always strive to do it better and with greater sensitivity to the human experiences of those who seek our care.

Dr. Aftab: What do you envision for the future of psychiatry? What opportunities lie ahead for us?

Dr. Summergrad: I think we will see an expansion of awareness of mental disorders globally. While it may seem counterintuitive to say this in the midst of a global pandemic, over the course of the last 80 years, the global burden of disease related to communicable diseases has fallen across much of the developed world and the burden of disease attributable to noncommunicable disease has increased. Psychiatric disorders are among the most frequent noncommunicable disorders and are increasing as a proportion of total illness burden. As such, the need for mental health-related care will increase dramatically over the next half century, if not longer.

Second, I think our understanding of neurobiology, the impact of medical disorders, and pathophysiology related to mechanisms such as inflammation in psychiatric disorders will increase. Likewise, our appreciation will grow for non-allelic influences on the genetics or heritability of psychiatric disorders. I don't think we have come near to tapping the effects of epigenetics on psychiatric illnesses, and that will become increasingly important.

I also think that over time, our understanding of particular neurobiological pathways and our ability to modulate these pathways will increase. How that will eventually yield the ability to treat patients with greater precision I don't know, but I expect that will occur. Over time, we may even learn enough to have a workable theory of mind and brain, but I am not sure that all of these mysteries will yield anytime soon, and for some of them, answers may have to come from other domains of human experience. 